\section{Estimativas da cobertura mamográfica no Estado de Goiás, Brasil}

\author{
Estimated mammogram coverage \\ in Goiás State, Brazil
}

\footnotetext{
1 Centro Regional de Ciências Nucleares do Centro-Oeste, Comissão Nacional de Energia Nuclear, Abadia de Goiás, Brasil.

2 Programa de Pós-graduação em Ciências da Saúde, Universidade Federal de Goiás, Goiânia, Brasil.

3 Instituto Nacional de Câncer, Rio de Janeiro, Brasil. ${ }^{4}$ Hospital das Clínicas, Universidade Federal de Goiás, Goiânia, Brasil.

5 Superintendência de Vigilância Sanitária e Ambiental de Goiás, Goiânia Brasil.

Correspondência R. S. Corrêa Centro Regional de Ciências Nucleares do Centro-Oeste, Comissão Nacional de Energia Nuclear.

BR-060 KM 174,5, Abadia de Goiás, GO 75345-000, Brasil. rcorrea@cnen.gov.br
}

\begin{abstract}
This cross-sectional study aimed to estimate mammogram coverage in the State of Goiás, Brazil, describing the supply, demand, and variations in different age groups, evaluating 98 mammography services as observational units. We estimated the mammogram rates by age group and type of health service, as well as the number of tests required to cover $70 \%$ and $100 \%$ of the target population. We assessed the association between mammograms, geographical distribution of mammography machines, type of service, and age group. Full coverage estimates, considering 100\% of women in the 40-69 and 50-69-year age brackets, were $61 \%$ and $66 \%$, of which the Brazilian Unified National Health System provided $13 \%$ and $14 \%$, respectively. To achieve $70 \%$ coverage, 43,424 additional mammograms would be needed. All the associations showed statistically significant differences $(p<0.001)$. We conclude that mammogram coverage is unevenly distributed in the State of Goiás and that fewer tests are performed than required.
\end{abstract}

Mamography; Breast Neoplasms; Health Services Coverage

\author{
Rosangela da Silveira Corrêa 1,2 \\ Ruffo Freitas-Júnior 2 \\ João Emílio Peixoto 1,3 \\ Danielle Cristina Netto Rodrigues 4 \\ Maria Eugênia da Fonseca Lemos 1 \\ Lucy Aparecida Parreira Marins 5 \\ Érika Aparecida da Silveira 2
}

\section{Introdução}

O câncer de mama apresenta elevada incidência e mortalidade em todo o mundo, representando um problema de saúde pública 1,2,3. O aumento da detecção de casos de câncer de mama e a consequente redução da mortalidade por esta causa no continente norte-americano e em países no norte da Europa têm sido atribuídos, em parte, à introdução do rastreamento organizado e ao tratamento adequado dos casos identificados 4,5 .

Atualmente, entre os métodos disponíveis para diagnóstico e detecção precoce do câncer de mama, o mais indicado para o rastreamento em massa é a mamografia. As razões para isso são: capacidade de mostrar todas as estruturas de importância para o diagnóstico, simplicidade do método e boa relação custo-efetividade 6 .

Ensaios clínicos aleatórios controlados evidenciaram redução significativa na mortalidade por câncer de mama, apontando os grupos etários beneficiados pelo rastreamento. Assim, houve impacto real sobre a mortalidade por esta doença em mulheres entre 50 e 69 anos que realizam mamografia a intervalos de dois anos. Nas mulheres mais jovens, na faixa etária entre $40 \mathrm{e}$ 49 anos, o resultado do estudo de maior casuística demonstrou impacto menor 7 .

Ao pesquisar a literatura, verifica-se que não há consenso quanto à faixa etária indicada para a realização da mamografia 8,9. Isso também ocor- 
re no Brasil, pois o Instituto Nacional de Câncer (INCA) recomenda a realização de mamografia bienal para mulheres na faixa etária de 50 a 69 anos 10, a Sociedade Brasileira de Mastologia (SBM) preconiza que mulheres acima de 40 anos devem realizar mamografia anualmente $11 \mathrm{e}$, a partir de 2009, a Lei Federal $n^{\circ}$. 11.664, de 29 de abril de 2008, assegura a realização de exame mamográfico pelo Sistema Único de Saúde (SUS) a todas as mulheres com 40 anos ou mais 12 .

A Organização Mundial da Saúde (OMS) sugere que, para o início de um programa de rastreamento mamográfico de base populacional, pelo menos $70 \%$ da população-alvo sejam cobertos pela mamografia 13 . No Brasil, programas de rastreamento organizado ainda não são realidade em decorrência de diversos fatores, como: falta de infraestrutura necessária para todas as rotinas do programa, baixo grau de cobertura da população-alvo com a mamografia, alto custo dos procedimentos e falta de seguimento da população estudada a médio e longo prazos. Por isso, são conduzidos somente rastreamentos oportunísticos, quando as mulheres procuram espontaneamente os serviços de saúde e recebem recomendações para que realizem o exame e, a seguir, são submetidas ao procedimento ${ }^{14}$.

Diante do exposto, este estudo teve como objetivo principal estimar a cobertura da mamografia, descrevendo a oferta e a demanda no Estado de Goiás, Brasil, e as variações de cobertura para as diversas faixas etárias. Em adição a isso, teve como objetivos secundários analisar a infraestrutura instalada e o tipo de atendimento oferecido às mulheres por regional de saúde no estado.

\section{Métodos}

Este estudo transversal teve como unidade de observação os serviços de mamografia no Estado de Goiás. Participaram da pesquisa 98 serviços, cujos mamógrafos estavam em uso em 2008 e que realizaram este tipo de exame para o SUS e para o sistema não SUS, que inclui o sistema de saúde suplementar (convênios de saúde) e as mulheres que pagaram o exame com recursos próprios. Foram excluídos do estudo os serviços cujos mamógrafos estavam fora de operação por problemas técnicos ou interditados pelos órgãos responsáveis por sua fiscalização e controle.

\section{Área de estudo}

O Estado de Goiás está localizado na Região Centro-Oeste do país, ocupando uma área de $340.103,467 \mathrm{~km}^{2}$ (Instituto Brasileiro de Geografia e Estatística. Estados@: Goiás. http://www.ibge. gov.br/estadosat/perfil.php?sigla=go, acessado em 18/Jun/2009), com população de 5.844.996 habitantes em 2008 15. Possui 246 municípios distribuídos em 16 regionais de saúde 16. O Município de Goiânia, capital do estado, fica localizado a $209 \mathrm{~km}$ da capital federal, Brasília.

\section{Levantamento dos dados de infraestrutura}

Os serviços de mamografia foram identificados a partir do Cadastro Nacional de Estabelecimentos de Saúde (CNES. http://cnes.datasus.gov. br, acessado em 12/Jan/2008) do Ministério da Saúde e, para o levantamento da infraestrutura disponível, foi aplicado instrumento de coleta de dados elaborado especificamente para esta pesquisa.

A localização geográfica dos serviços incluídos na pesquisa foi estabelecida utilizando-se o Plano Diretor de Regionalização de Goiás, que divide o estado em regionais de saúde 16 .

As informações acerca da população feminina por faixa etária, de acordo com as regionais de saúde, foram obtidas na página na Internet do Departamento de Informática do SUS (DATASUS. Informações de saúde: projetos intercensitários 2008. http://tabnet.datasus.gov. $\mathrm{br} / \mathrm{cgi} /$ deftohtm.exe?ibge/cnv/popgo.def, acessado em 12/Jul/2009), com base nos dados intercensitários do Instituto Brasileiro de Geografia e Estatística (IBGE) para 2008.

\section{População-alvo e cobertura}

Neste estudo, as mulheres na faixa etária de 40 a 69 anos e aquelas com 35 anos ou mais apresentando risco elevado para câncer de mama foram consideradas como população-alvo. A estimativa da cobertura foi expressa em porcentagem, entre o número de exames realizados pelo número dos exames esperados na população-alvo.

Para o cálculo do número de exames esperados desta população foi utilizada a recomendação do INCA, órgão oficial do Ministério da Saúde para o desenvolvimento das políticas de saúde para o país 17 , ajustada para a faixa etária de 40 a 49 anos. Na programação de procedimentos, faz-se necessário prever que, em determinado ano, $50 \%$ das mulheres na faixa etária de 50 a 69 anos farão rastreamento por meio do exame clínico da mama, complementado com mamografia diagnóstica em 8,9\% desta população, que apresentarão exame clínico da mama alterado, enquanto os outros $50 \%$ realizarão exame clínico da mama e mamografia de rastreamento, independentemente dos achados no exame clínico da mama. No grupo de risco ele- 
vado para câncer de mama, a base populacional equivale a $1 \%$ das mulheres de 35 anos ou mais, entre as quais, estima-se que $100 \%$ farão mamografia de rastreamento e 8,9\% farão mamografia diagnóstica 17 .

Para a estimativa da cobertura do SUS, os exames realizados referem-se aos dados da produção mamográfica da população-alvo disponíveis no Sistema de Informações Ambulatoriais (SIA) do DATASUS (http://tabnet.datasus.gov.br/cgi/ tabcgi.exe?sia/cnv/qbgo.def, acessado em 10/ Jul/2009). Os dados referentes à produção mamográfica do sistema não SUS foram informados pelos próprios serviços incluídos no estudo.

Foram calculadas as frequências de realização da mamografia pelo SUS para as faixas etárias de menos de 35 anos, de 35 a 39 anos, de 40 a 49 anos, de 50 a 59 anos, de 60 a 69 anos e de 70 anos ou mais. Como para os dados sobre o sistema não SUS coletados não havia informações sobre as faixas etárias, estes foram ajustados considerando-se para este estudo a mesma distribuição por faixa etária dos exames realizados pelo SUS. Assim, a partir desses dados, foram avaliadas as estimativas da cobertura pelo SUS e pelo sistema não SUS para 70\% 13 e 100\% da população-alvo.

\section{Análise de dados}

A análise estatística foi realizada por meio do programa SPSS (SPSS Inc., Chicago, Estados Unidos). Foram calculadas as frequências absolutas e relativas das variáveis e os percentuais de cobertura com intervalo de 95\% de confiança (IC95\%). Utilizou-se o teste qui-quadrado para avaliar a associação entre a realização da mamografia e a distribuição geográfica dos mamógrafos, o tipo de atendimento e a faixa etária, adotando-se o nível de significância de 5\%.

Considerando-se que cada serviço pode realizar trinta exames diários, as regionais de saúde foram categorizadas de acordo com sua capacidade potencial de realização de mamografias anualmente, bienalmente ou acima de dois anos, conforme a população-alvo, em: categoria 1 (um mamógrafo para 6.000 mulheres ou menos); categoria 2 (um mamógrafo para a faixa de 6.001 a 12.000 mulheres); e categoria 3 (um mamógrafo para 12.001 ou mais mulheres).

\section{Aspectos éticos}

Este estudo foi aprovado pelo Comitê de Ética em Pesquisa "Dr. Henrique Santillo", da Secretaria de Estado de Saúde de Goiás, protocolo no. 00061177000-5, não tendo sido necessário assinar Termo de Consentimento Livre e Esclarecido.

\section{Resultados}

Em 2008, dos 115 mamógrafos existentes no Estado de Goiás, 103 estavam em uso, instalados em 98 serviços. Desse total de serviços, 45 $(45,9 \%)$ participavam do atendimento ao SUS e $53(54,1 \%)$ faziam atendimento exclusivamente para o sistema não SUS. Entre os 45 serviços que atendiam ao SUS, 10 (22,2\%) eram públicos e 35 (77,8\%) pertenciam a serviços privados com credenciamento do SUS para mamografia.

Pela análise das características tecnológicas e dos anos de operação dos 103 mamógrafos em uso, constatou-se que 78 unidades $(75,7 \%)$ eram do tipo convencional, em que a imagem é adquirida e registrada em filme de raios- $\mathrm{X}$, enquanto 25 (24,3\%) eram do tipo digital, no qual a imagem é adquirida com o auxílio de técnicas numéricas em detectores específicos e registrada em um sistema computadorizado. Desse total, $83(80,6 \%)$ mamógrafos eram importados e 20 (19,4\%), de fabricação nacional, 68 (66\%) estavam em uso há 15 anos ou menos e 35 (34\%), há mais de 15 anos.

Os mamógrafos em uso no estado encontravam-se distribuídos em 31 municípios e todas as 16 regionais de saúde possuíam pelo menos um equipamento, embora duas delas não possuíssem mamógrafos que atendessem ao SUS. Em relação à distribuição geográfica dos mamógrafos em uso, na Tabela 1, observa-se que 51 $(49,5 \%)$ estavam instalados na regional de saúde Central e nove $(8,7 \%)$ na regional Pirineus, estando os restantes $43(41,8 \%)$ distribuídos entre as demais regionais de saúde.

No ano de 2008, entre a população residente no Estado de Goiás, havia 2.952.975 mulheres, das quais, $790.770(26,7 \%)$ estavam na faixa etária de 40 a 69 anos e 402.489 (13,6\%), de 50 a 69 anos. Para as faixas etárias de 40 a 69 anos e de 50 a 69 anos, respectivamente, estimaram-se para o Estado de Goiás as relações de 7.677 mulheres/mamógrafo e 3.908 mulheres/mamógrafo, variando de 4.688 mulheres/mamógrafo e 2.444 mulheres/mamógrafo na regional Central a 17.828 mulheres/mamógrafo e 9.596 mulheres/ mamógrafo na regional Oeste I. A distribuição do número de mulheres para as duas faixas etárias estudadas por regional de saúde encontrase na Tabela 1 .

Na Figura 1, são apresentadas as regionais de saúde de acordo com a capacidade potencial de realização de mamografias em mulheres nas faixas de 40 a 69 anos. Nessa faixa etária, uma regional de saúde do Estado de Goiás estava na categoria 1, sete na categoria 2 e as demais, na categoria 3. Para a faixa etária de 50 a 69 anos, nove regionais de saúde estavam na categoria 1 : 
Distribuição geográfica dos mamógrafos em uso por regional de saúde e das mulheres nas faixas etárias de 40 a 69 anos e de 50 a 69 anos. Estado de Goiás, Brasil, 2008.

\begin{tabular}{|c|c|c|c|c|}
\hline \multirow[t]{2}{*}{ Regional } & \multicolumn{2}{|c|}{ Mulheres } & \multicolumn{2}{|c|}{ Mamógrafos } \\
\hline & $40-69$ anos & $50-69$ anos & Total & SUS \\
\hline Central & 239.095 & 124.669 & 51 & 16 \\
\hline Centro-sul & 97.890 & 46.806 & 5 & 3 \\
\hline Entorno Norte & 32.039 & 15.686 & 4 & 2 \\
\hline Entorno Sul & 65.220 & 28.022 & 5 & 3 \\
\hline Estrada de ferro & 36.628 & 19.282 & 3 & 1 \\
\hline Nordeste & 6.907 & 3.655 & 1 & 1 \\
\hline Norte & 18.269 & 9.573 & 2 & 2 \\
\hline Oeste I & 17.828 & 9.596 & 1 & 1 \\
\hline Oeste II & 16.414 & 8.911 & 1 & 0 \\
\hline Pirineus & 66.261 & 34.429 & 9 & 5 \\
\hline Rio Vermelho & 27.998 & 14.675 & 4 & 2 \\
\hline São Patrício & 41.208 & 21.940 & 3 & 2 \\
\hline Serra da Mesa & 15.512 & 8.142 & 1 & 0 \\
\hline Sudoeste I & 48.271 & 24.755 & 4 & 2 \\
\hline Sudoeste II & 25.419 & 13.239 & 4 & 2 \\
\hline Sul & 35.811 & 19.109 & 5 & 3 \\
\hline Goiás & 790.770 & 402.489 & 103 & 45 \\
\hline
\end{tabular}

SUS: Sistema Único de Saúde.

Fonte: dados populacionais (Departamento de Informática do SUS. Informações de saúde: projetos intercensitários 2008. http://tabnet.datasus.gov.br/cgi/deftohtm.exe?ibge/cnv/popgo.def, acessado em 12/Jul/2009); dados sobre o número de mamógrafos originais dos autores.

Norte, Nordeste, Rio Vermelho, Entorno Norte, Pirineus, Central, Entorno Sul, Sudoeste II e Sul; sete na categoria 2: Serra da Mesa, São Patrício, Oeste I, Oeste II, Centro Sul, Estrada de Ferro e Sudoeste I e; nenhuma na categoria 3.

O número de exames esperados anualmente para $100 \%$ e $70 \%$ de cobertura em 2008 , de acordo com as faixas etárias incluídas neste estudo, é apresentado na Tabela 2. Esperava-se a realização de 231.524 exames para a cobertura de $100 \%$ e de 165.777 exames para $70 \%$ de cobertura da população feminina entre 50 e 69 anos e das mulheres com risco elevado para câncer de mama. Para a faixa de 40 a 69 anos e o grupo de risco seriam esperados 442.942 exames para $100 \%$ de cobertura e 313.770 para $70 \%$.

Em 2008, foram realizadas 59.462 mamografias pelo SUS no Estado de Goiás, tendo sido $59.144(99,5 \%)$ para o sexo feminino e $318(0,5 \%)$ para o sexo masculino. Das mamografias realizadas em mulheres, $1.655(2,8 \%)$ foram na faixa etária abaixo de 35 anos, 4.532 (7,7\%) entre 35 e 39 anos, 24.826 (42\%) entre 40 e 49 anos, 18.201 $(30,8 \%)$ entre 50 e 59 anos, 7.472 (12,6\%) entre 60 e 69 anos e $2.458(4,1 \%)$ naquelas com 70 anos ou mais. Os coeficientes de realização de mamografias pelo SUS nas faixas etárias de 40 a 49 anos, 50 a 59 anos e 60 a 69 anos foram 64, 72 e 50 por mil mulheres, respectivamente. Em relação à produção por natureza do serviço, 46.673 (78,5\%) mamografias foram realizadas em estabelecimentos privados, 8.362 (14\%) em serviços filantrópicos e 4.427 (7,45\%) pela rede própria. O sistema não SUS realizou 212.857 mamografias para mulheres na faixa de 40 a 69 anos de idade e no grupo de risco.

Na Tabela 3, observa-se desigualdade tanto na distribuição da realização de exames entre as categorias das regionais de saúde quanto no número de mamografias realizadas pelo SUS e pelo sistema não SUS, tendo este último realizado quatro vezes mais exames do que o primeiro $(\mathrm{p}<0,001)$.

Para atender $100 \%$ e $70 \%$ da população feminina de 40 a 69 anos no Estado de Goiás, seria necessário realizar 172.594 e 43.424 mamografias adicionais ao ano, respectivamente. Houve variação significativa na taxa percentual de cobertura mamográfica para $100 \%$ da população-alvo entre as diversas regionais de saúde do Estado de 
Figura 1

Representação espacial da relação de mulheres de 40 a 69 anos por mamógrafo em uso de acordo com as regionais de saúde. Estado de Goiás, Brasil, 2008.

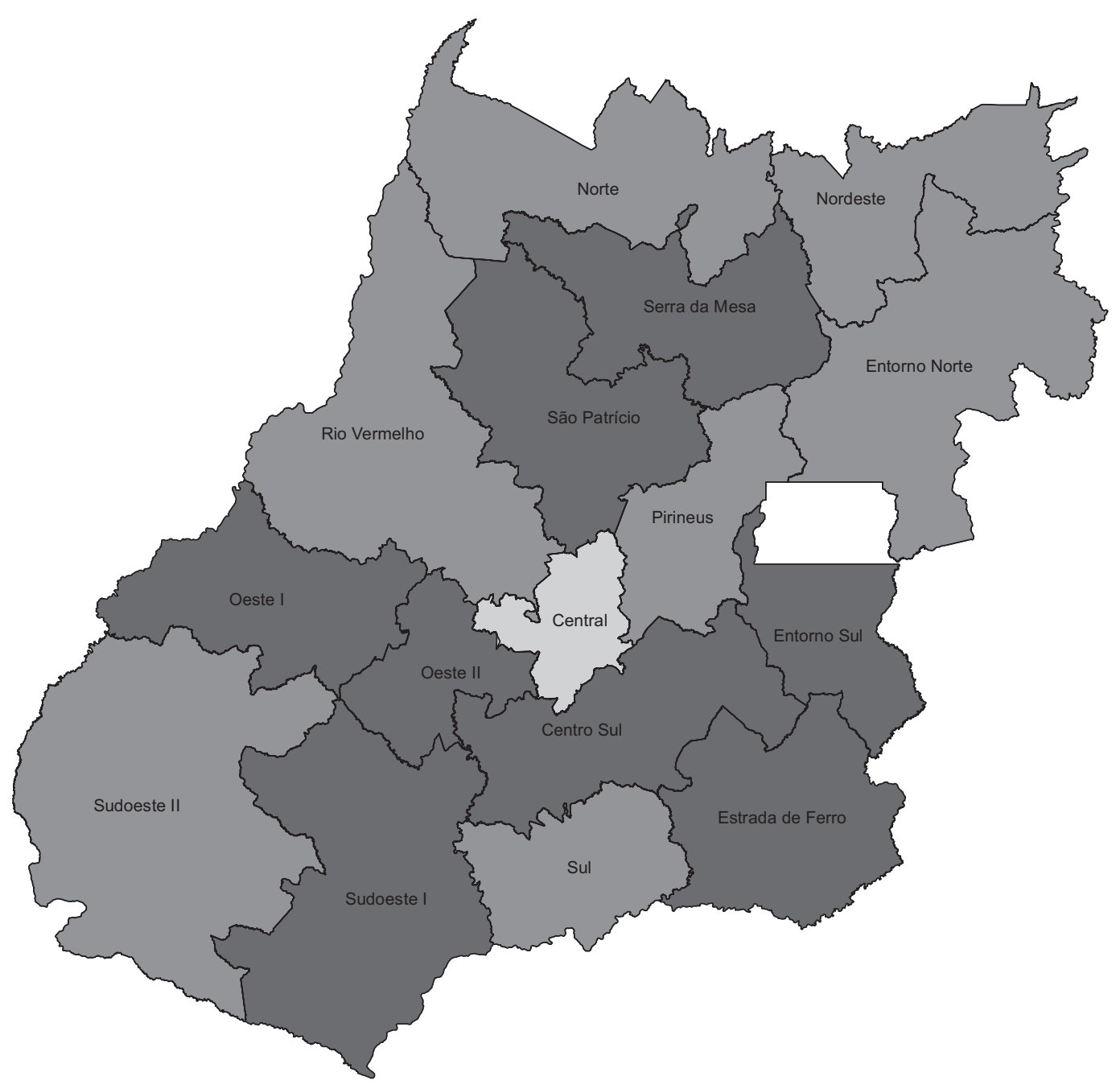

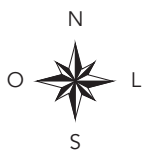

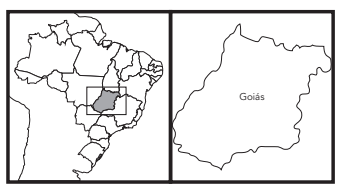

Mulheres de 40 a 69 anos/mamógrafos

$\square$ Categoria 1: 6.000 ou menos

Categoria 2: 6.001 a 12.000

Categoria 3: 12.001 ou mais 
Estimativa de mamografias para cobertura de 100\% e de 70\% da população-alvo. Estado de Goiás, Brasil, 2008.

\begin{tabular}{lccc}
\hline População-alvo & \multicolumn{2}{c}{ Tipo de mamografia } \\
& Rastreamento & Diagnóstico & Total \\
& & & \\
\hline 100\% cobertura bienal & 395.385 & 35.189 & 430.574 \\
$40-69$ anos & 201.245 & 17.911 & 219.156 \\
$50-69$ anos & 11.357 & 1.011 & 12.368 \\
Grupo de risco * & & & 301.402 \\
$70 \%$ cobertura bienal & 276.770 & 24.632 & 153.409 \\
$40-69$ anos & 140.871 & 12.538 & 12.368 \\
50-69 anos & 11.357 & 1.011 & \\
Grupo de risco & &
\end{tabular}

* Mulheres com 35 anos ou mais apresentando risco elevado para câncer de mama.

Fonte: Dados populacionais (Departamento de Informática do SUS. Informações de saúde: projetos intercensitários 2008. http://tabnet.datasus.gov.br/cgi/deftohtm.exe?ibge/cnv/popgo.def, acessado em 12/Jul/2009); forma de cálculo baseada em parâmetros do Instituto Nacional de Câncer 17.

Tabela 3

Número total de mamografias (Sistema Único de Saúde - SUS e não SUS) realizadas por categoria das regionais de saúde e por tipo de atendimento, número de mamografias realizadas pelo SUS por faixa etária e comparações estatísticas. Estado de Goiás, Brasil, 2008.

\begin{tabular}{lccc}
\hline Variável & Exames realizados $(\mathbf{n})$ & $\begin{array}{c}\text { Mamografia } \\
\text { População-alvo (\%) }\end{array}$ & Valor de $\mathbf{p}$ * \\
\hline $\begin{array}{l}\text { Capacidade potencial de realização } \\
\text { de mamografias }\end{array}$ & & & \\
$\quad$ Categoria 1 & 179.690 & 134,2 & \\
Categoria 2 & 45.788 & 38,4 & $<0,001$ \\
Categoria 3 & 44.868 & 23,6 & $<0,001$ \\
Tipo de atendimento & & & \\
SUS & 57.489 & 13,0 & \\
Sistema não SUS & 212.857 & 48,0 & $<0,001$ \\
Faixa etária (anos) [SUS] & & 10,9 & \\
40-49 & 24.826 & 12,2 & \\
50-59 & 18.201 & 8,6 & 11,9 \\
60-69 & 7.472 & 6.990 & \\
Grupo de risco ** & & & \\
\hline
\end{tabular}

* Teste qui-quadrado;

** Mulheres com 35 anos ou mais apresentando risco elevado para câncer de mama.

Nota: categoria 1 (um mamógrafo para 6.000 mulheres ou menos); categoria 2 (um mamógrafo para a faixa de 6.001 a 12.000 mulheres); categoria 3 (um mamógrafo para 12.001 ou mais mulheres)

Fonte: dados do SUS no Sistema de Informações Ambulatoriais (SIA) do Departamento de Informática do SUS (DATASUS. http://tabnet.datasus.gov.br/cgi/tabcgi.exe?sia/cnv/qbgo.def, acessado em 10/Jul/2009); dados do sistema não SUS originais dos autores. 
Goiás, desde 2,7\% na regional Oeste I até 134,2\% na regional Central.

A distribuição percentual da cobertura (SUS e não SUS) para $100 \%$ da população-alvo definida neste estudo, de acordo com as regionais de saúde, estão apresentados na Figura 2. Para a faixa etária de 40 a 69 anos, a estimativa da cobertura no Estado de Goiás foi de $61 \%$, tendo o SUS contribuído com 13\% e o sistema não SUS, com $48 \%$. Para a faixa etária de 50 a 69 anos, a estimativa da cobertura no Estado de Goiás foi de $66 \%$, tendo o SUS contribuído com $14 \%$ e o sistema não SUS, com 52\%. Embora a regional Nordeste disponha de um mamógrafo da rede própria do SUS, no SIA do DATASUS não foram encontradas informações sobre o número de exames realizados em 2008.

\section{Discussão}

A cobertura mamográfica como indicador de acesso pode ser avaliada a partir do número de mamógrafos existentes no estado, de sua distribuição geográfica e capacidade operacional 18 . Considerando que cada mamógrafo pode realizar até trinta exames diários em um total de 400 dias úteis em dois anos, é possível estabelecer que, neste período, cada mamógrafo poderia atender a 12.000 mulheres 19. Assim, seria possível pressupor que Goiás dispõe de um número de mamógrafos suficiente para atender à população de 790.770 mulheres, entre 40 e 69 anos para realizar mamografia bienalmente. Porém, a análise desse parâmetro por regional de saúde mostra que a suficiência é apenas aparente, tanto em função da distribuição desigual dos mamógrafos como também pela baixa produção de exames pela rede própria do SUS, o que se reflete na infraestrutura de atendimento às mulheres. Entretanto, 35 mamógrafos instalados e em funcionamento têm mais de 15 anos de fabricação, o que indica que, em breve, deverão ser substituídos por novos aparelhos com tecnologia mais moderna e adequada.

Para assegurar a base populacional de um programa de rastreamento organizado, este deve ser implantado pelo setor público e as mulheres atendidas pelo setor privado devem ser elegíveis para nele participar 20 . Assim, ao comparar os indicadores de cobertura em relação à base populacional, constata-se que a participação do SUS na cobertura mamográfica do Estado de Goiás ainda é pequena, acompanhando a mesma tendência no país como um todo 21,22 . Isso pode ser decorrente da distribuição desigual dos mamógrafos e de sua capacidade operacional reduzida, refletindo-se nos níveis de cobertura estimados por regional de saúde. Como instrumento de equidade social em um programa de detecção precoce do câncer de mama, a participação do governo federal, por intermédio do SUS, torna-se primordial e pode interferir diretamente no que se espera de um programa equânime, integral e de fácil acesso.

Corroborando os resultados encontrados no presente estudo para o Estado de Goiás, nos Estados Unidos, país em que a American Cancer Society 23 recomenda a mamografia a partir dos 40 anos para todas as mulheres, também foi constatada desigualdade na cobertura mamográfica em 2006, variando de 48,7\% a 71,4\% nos vários estados avaliados.

Comparando-se os dados apurados no presente estudo para a faixa etária de 40 a 69 anos com os dados obtidos na Pesquisa Nacional por Amostra de Domicílios (PNAD) em 2003 22, observa-se que a cobertura mamográfica no Estado de Goiás aumentou de $48 \%$ para $61 \%$. Na faixa etária de 50 a 69 anos, a prevalência de realização de mamografias, de acordo com a PNAD, foi de $46 \%$ em 2003 22, aumentando para $71 \%$ em 2008 24, sendo este último valor próximo ao encontrado neste estudo, que registrou $66 \%$. No entanto, a mesma comparação mostra que não houve mudança percentual na cobertura mamográfica pelo SUS, uma vez que o aumento verificado se deu em virtude do crescimento da cobertura instituída pelo sistema não SUS no período.

Deve-se destacar, entretanto, que há diferenças entre a metodologia empregada na PNAD de 2003 e 2008 e a que foi utilizada na pesquisa aqui apresentada. Enquanto na PNAD foi utilizada metodologia baseada em inquérito domiciliar realizado com uma parcela da população, no presente trabalho, foram levantados os números médios de exames mensais realizados pelos serviços de mamografia no Estado de Goiás no ano de 2008.

Ainda que esses estudos não sejam plenamente comparáveis, um dos fatores sugestivos do aumento da cobertura mamográfica no Estado de Goiás é a redução do número de casos avançados de tumores, concomitantemente ao aumento expressivo do número de tumores de mama iniciais não invasivos observados pelo Registro de Câncer de Base Populacional de Goiânia 25,26. Isso explica, pelo menos em parte, que o rastreamento mamográfico oportunístico tem conseguido detectar um número maior de casos, fato também observado previamente em outros locais que utilizaram a mamografia como método de rastreamento nos anos 80 e 90 2,27.

Existem controvérsias, no Brasil e em outros países, a respeito da faixa etária em que deve ser iniciado o rastreamento mamográfico e qual o 
Distribuição percentual da cobertura de mamografia para mulheres de 40 a 69 anos, 50 a 69 anos, por tipo de atendimento e por regional de saúde. Estado de Goiás, Brasil, 2008.

2a) Mulheres de 40 a 69 anos

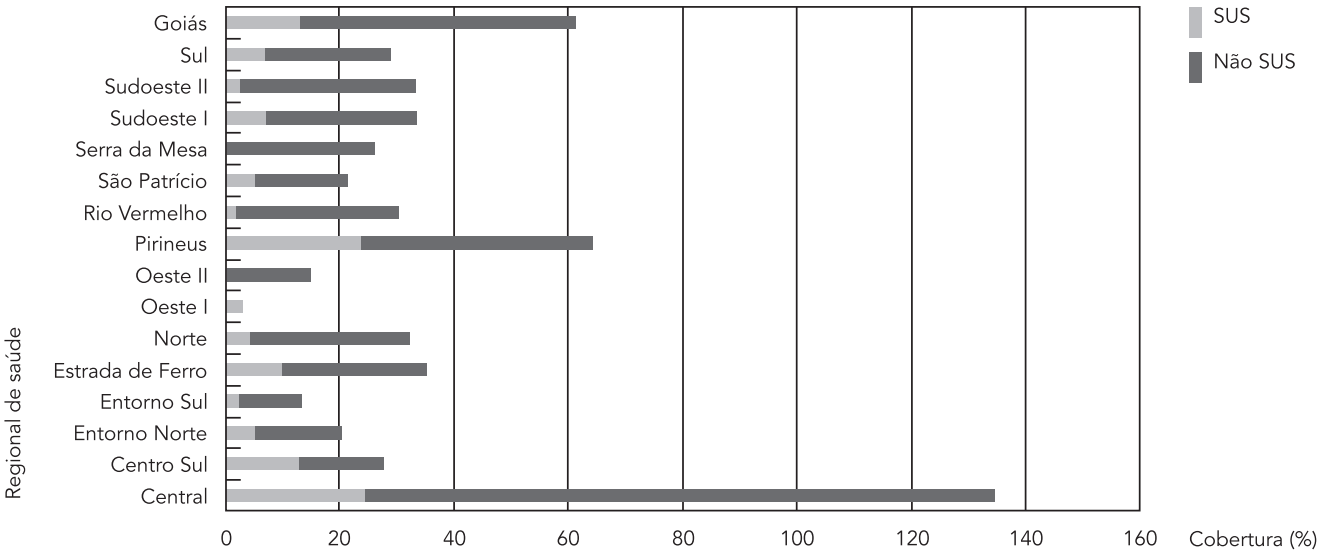

2b) Mulheres de 50 a 69 anos

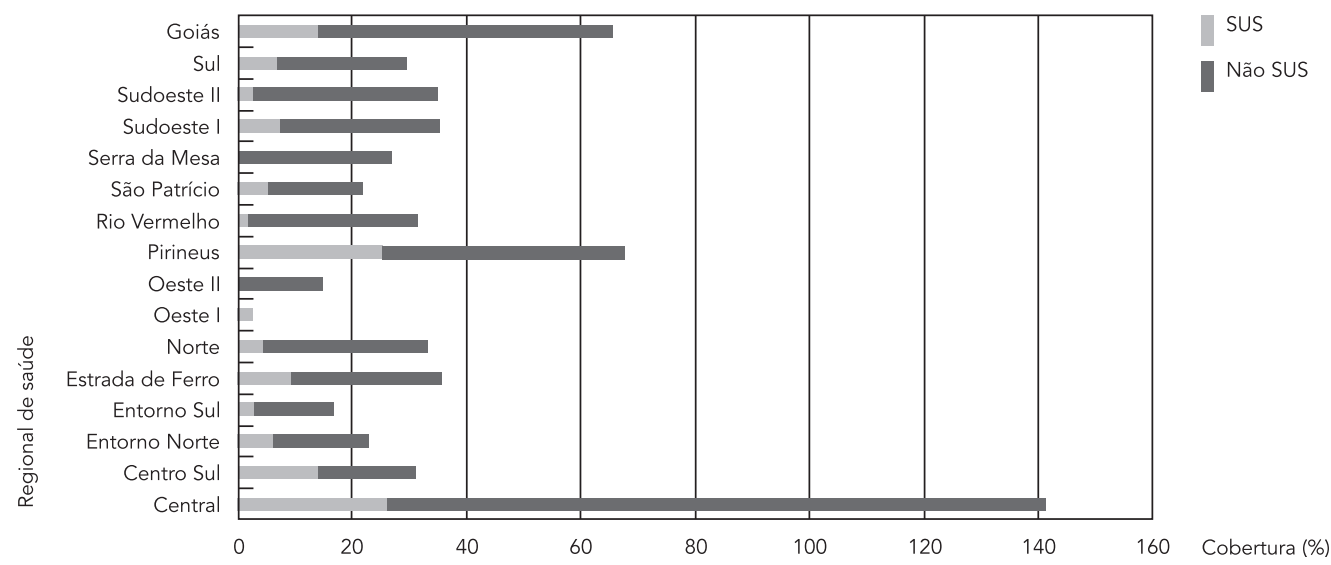

Nota: na regional central o número de exames realizados pelos serviços foi superior ao número de exames esperados de acordo com a população-alvo.

melhor intervalo para realizá-lo 8,9. Embora haja essa ausência de consenso entre organismos nacionais 10,11,12, analisando os dados da produção da mamografia pelo SUS em 2008, por faixa etária, verificou-se prevalência de mamografias no grupo de 40 a 49 anos. Esses resultados se mostram consistentes com as observações de outros estudos, nos quais se identificou a relação médico-paciente como o preditor mais forte para a realização da mamografia nesta faixa etária 28,29 .
Uma limitação do nosso estudo consiste no fato de ser um rastreamento oportunístico, em que não há controle sobre a população que realiza a mamografia. Assim, pode ocorrer repetição de exames em uma mesma mulher, o que pode levar a resultados superestimados. Todavia, nossos resultados se mostraram coerentes com os de outros estudos 22,23,24.

A análise por regional de saúde mostrou que, com exceção da regional Central, a qual apresentou cobertura de $134 \%$, e da regional Pirineus 
com $64 \%$ de cobertura, as demais regionais apresentaram valores abaixo daqueles registrados pela PNAD em 200322 e em 2008 24, tanto para exames realizados pelo SUS quanto para os não SUS. Portanto, o Estado de Goiás deve rever suas políticas de saúde e aparelhar melhor suas próprias unidades de saúde, além de realizar ações que melhorem o desempenho dos programas de assistência à saúde da mulher.

Na Constituição Brasileira de 1988, a saúde é colocada como um direito de todos e um dever do Estado. Trata-se de uma questão de justiça social, de direitos humanos fundamentais e de promoção da equidade em matéria de saúde. Contudo, essa é uma área, como tantas outras, em que a teoria e a prática estão muitas vezes dissociadas.

\section{Conclusão}

Os resultados aqui apresentados mostram que a estimativa da cobertura mamográfica é desigual entre as regionais de saúde do Estado de Goiás. Nessa perspectiva, detectou-se que a regional Central possui uma grande concentração de mamógrafos e realiza um número de exames superior da população-alvo em comparação com as demais regionais de saúde. Também se observou que a oferta de exames é menor do que a demanda, que a produção do SUS é inferior à do sistema não SUS e que há prevalência de mamografias pelo SUS no grupo de mulheres entre 40 e 49 anos. Por fim, o número de mamógrafos em uso é suficiente, embora haja necessidade de substituição de um quarto deles nos próximos anos.

\section{Resumo}

Este estudo transversal objetivou estimar a cobertura da mamografia no Estado de Goiás, Brasil, descrevendo sua oferta, demanda e variações para as diversas faixas etárias, tendo como unidades de observação 98 serviços de mamografia. Foram estimados as frequências de realização da mamografia por faixa etária e tipo de sistema de saúde, bem como o número de exames necessários para a cobertura de 70\% e 100\% da população-alvo. Foi avaliada a associação entre a realização da mamografia, a distribuição geográfica dos mamógrafos, o tipo de atendimento e a faixa etária. As estimativas de cobertura total para $100 \%$ das mu- lheres nas faixas etárias de 40-69 anos e de 50-69 anos foram de $61 \%$ e 66\%, tendo o Sistema Único de Saúde contribuído com $13 \%$ e $14 \%$, respectivamente. Para atingir $70 \%$ de cobertura, seria necessário realizar 43.424 mamografias adicionais. Todas as associações apresentaram diferença estatística significativa $(p<$ 0,001). Conclui-se que a cobertura da mamografia está distribuída de maneira desigual no Estado de Goiás e o número de exames realizados é inferior ao necessário.

Mamografia; Neoplasias de Mama; Cobertura de Serviços de Saúde 


\section{Colaboradores}

R. S. Corrêa colaborou na concepção, aquisição e análise dos dados, redação do artigo e aprovação final. R. Freitas-Júnior e E. A. Silveira participaram da análise e interpretação dos dados, revisão crítica do artigo e aprovação final. J. E. Peixoto contribuiu na concepção, análise e interpretação dos dados revisão crítica do artigo e aprovação final. D. C. N. Rodrigues colaborou na aquisição de dados, análise e interpretação dos dados, redação do artigo e aprovação final. M. E. F. Lemos e L. A. P. Marins contribuíram na aquisição dos dados, revisão crítica e aprovação.

\section{Referências}

1. Caleffi M, Ribeiro RA, Duarte Filho DL, AshtonProlla P, Bedin Junior AJ, Skonieskil GP, et al. A model to optimize public health care and downstage breast cancer in limited-resource populations in southern Brazil. (Porto Alegre Breast Health Intervention Cohort). BMC Public Health 2009; 9:83.

2. Parkin DM, Whelan SL, Ferlay J, Teppo L, Thomas DB. Cancer incidence in five continents. Lyon: International Agency for Research on Cancer; 2002.

3. Instituto Nacional de Câncer. Estimativa 2010: incidência de câncer no Brasil. Rio de Janeiro: Instituto Nacional de Câncer; 2009.

4. Jemal A, Center MM, DeSantis C, Ward EM. Global patterns of cancer incidence and mortality rates and trends. Cancer Epidemiol Biomarkers Prev 2010; 19:1893-907.

5. Smith RA, Cokkinides V, Brawley OW. Cancer screening in the United States, 2009: a review of current American Cancer Society guidelines and issues in cancer screening. CA Cancer J Clin 2009; 59:27-41.

6. Perry N, Broeders M, Wolf C, Törnberg S, Holland R, von Karsa L. European guidelines for quality assurance in breast cancer screening and diagnosis. $4^{\text {th }}$ Ed. Brussels: European Commission; 2006.

\section{Agradecimentos}

Aos servidores do Centro Regional de Ciências Nucleares do Centro-Oeste, Comissão Nacional de Energia Nuclear (CRCN-CO/CNEN) e da Superintendência de Vigilância Sanitária e Ambiental de Goiás que colaboraram com a coleta de dados. E ao Instituto Avon pelo apoio financeiro para a revisão do trabalho. $\mathrm{O}$ trabalho foi financiado pelo Edital PPSUS 2004 (Departamento de Ciência e Tecnologia do Ministério da Saúde DECIT/MS) por intermédio do Conselho Nacional de Desenvolvimento Científico e Tecnológico (CNPq) e apoio da Secretaria de Ciência e Tecnologia do Estado de Goiás (SECTEC-GO).
7. Autier P, Boniol M, La Vecchia C, Vatten L, Gavin A, Héry C, et al. Disparities in breast cancer mortality trends between 30 European countries: retrospective trend analysis of WHO mortality database. BMJ 2010; 341:c3620.

8. U.S. Preventive Services Task Force. Screening for breast cancer: U.S. Preventive Services Task Force recommendation statement. Ann Intern Med 2009; 151:716-26.

9. Nelson HD, Tyne K, Naik A, Bougatsos C, Chan BK, Humphrey L, et al. Screening for breast cancer: an update for the U.S. Preventive Services Task Force. Ann Intern Med 2009; 151:727-37.

10. Instituto Nacional de Câncer. Controle do câncer de mama: documento de consenso. Rio de Janeiro: Instituto Nacional de Câncer; 2004.

11. Sociedade Brasileira de Mastologia. Recomendações da X Reunião Nacional de Consenso: rastreamento do câncer de mama na mulher brasileira. http://www.sbmastologia.com.br/downloads/ reuniao_de_consenso_2008.pdf (acessado em 03/ $\mathrm{Jul} / 2009$ ). 
12. Brasil. Lei no ${ }^{\circ}$ 11.664, de 29 de abril de 2008. Dispõe sobre a efetivação de ações de saúde que assegurem a prevenção, a detecção, o tratamento e o seguimento dos cânceres do colo uterino e de mama, no âmbito do Sistema Único de Saúde - SUS. Diário Oficial da União 2008; 30 abr.

13. World Health Organization. Early detection. Cancer control: knowledge into action. WHO guide for effective programmes. Module 3. Geneva: World Health Organization; 2007.

14. Instituto Nacional de Câncer. A situação do câncer no Brasil. Rio de Janeiro: Instituto Nacional de Câncer; 2006.

15. Instituto Brasileiro de Geografia Estatística. Estimativas das populações dos municípios em 2008. http://www.ibge.gov.br/home/presidencia/notici as/noticia_impressao.php?id_noticia=1215 (acessado em 20/Jun/2009).

16. Secretaria de Estado da Saúde, Governo do Estado de Goiás. Plano diretor de regionalização. 2a Ed. Goiânia: Superintendência de Planejamento; 2004.

17. Instituto Nacional de Câncer. Parâmetros técnicos para o rastreamento do câncer de mama: recomendações para gestores estaduais e municipais. Rio de Janeiro: Instituto Nacional de Câncer; 2009.

18. Instituto Nacional de Câncer. Inquérito domiciliar sobre comportamentos de risco e morbidade referida de doenças e agravos não transmissíveis: Brasil, 15 capitais e Distrito Federal 2002-2003. Rio de Janeiro: Instituto Nacional de Câncer; 2004.

19. Koch HA, Peixoto JE, Neves ALE. Análise da infraestrutura para a mamografia no Brasil. Radiol Bras 2000; 33:23-30.

20. Corrêa RS, Peixoto JE, Silver LD, Dias CM, Nogueira MS, Hwang SF, et al. Impacto de um programa de avaliação da qualidade da imagem nos serviços de mamografia do Distrito Federal. Radiol Bras 2008; 41:109-14.

21. Instituto Nacional de Câncer. Encontro internacional sobre rastreamento de câncer de mama. http://www2.inca.gov.br/wps/wcm/connect/d05 766004e85afde866a9f56664898a3/recomenda coes_encontro_internacional_rastreamento_can cer_de_mama.pdf?MOD=AJPERES\&CACHEID= d05766004e85afde866a9f56664898a3 (acessado em 10/Dez/2009).
22. Instituto Brasileiro de Geografia e Estatística. Pesquisa Nacional por Amostra de Domicílios: acesso e utilização de serviços de saúde 2003. Rio de Janeiro: Instituto Brasileiro de Geografia e Estatística; 2005.

23. American Cancer Society. Cancer prevention and early detection. Facts and figures, 2009. Atlanta: American Cancer Society; 2009.

24. Instituto Brasileiro de Geografia e Estatística. Pesquisa Nacional por Amostra de Domicílios: um panorama da saúde no Brasil. Acesso e utilização dos serviços, condições de saúde e fatores de risco e proteção à saúde - 2008. Rio de Janeiro: Instituto Brasileiro de Geografia e Estatística; 2010.

25. Martins E, Freitas-Júnior R, Curado MP, Freitas NM, Oliveira JC, Silva CM. Evolução temporal dos estádios do câncer de mama ao diagnóstico em um registro de base populacional no Brasil Central. Rev Bras Ginecol Obstet 2009; 31:219-23.

26. Freitas-Júnior R, Freitas NM, Curado MP, Martins E, Moreira MA, Silva CM. Variations in breast cancer incidence per decade of life (Goiânia, GO, Brazil): 16-year analysis. Cancer Causes Control 2008; 19:681-7.

27. Shapiro BS, Venet W, Strax P, Venet L. Periodic screening for breast cancer: the health insurance plan project and its sequelae, 1963-1986. Baltimore: Johns Hopkins University Press; 1988.

28. Rauscher GH, Hawley ST, Earp JA. Baseline predictors of initiation vs. maintenance of regular mammography use among rural women. Prev Med $2005 ; 40: 822-30$

29. Lima-Costa MF, Matos DL. Prevalência e fatores associados à realização da mamografia na faixa etária de 50-69 anos: um estudo baseado na Pesquisa Nacional por Amostra de Domicílios (2003). Cad Saúde Pública 2007; 23:1665-73.

Recebido em 13/Set/2010

Versão final reapresentada em 12/Abr/2011

Aprovado em 19/Mai/2011 